\title{
Survival and Prognosis of Patients with Pilocytic Astrocytoma: A Single-Center Study
}

\author{
Jae Hui Park ${ }^{1}$, Nani Jung ${ }^{1}$, Seok Jin Kang ${ }^{1}$, Heung Sik Kim ${ }^{1}$, El Kim², Hee Jung Lee ${ }^{3}$, Hye Ra Jung ${ }^{4}$, Misun Choe ${ }^{4}$, Ye Jee Shim \\ Departments of ${ }^{1}$ Pediatrics, ${ }^{2}$ Neurosurgery, ${ }^{3}$ Radiology, ${ }^{4}$ Pathology, Keimyung University School of Medicine, \\ Keimyung University Dongsan Medical Center, Daegu, Korea
}

Received March 18, 2019

Revised June 24, 2019

Accepted July 12, 2019

\section{Correspondence}

Ye Jee Shim

Department of Pediatrics,

Keimyung University School of Medicine,

1095 Dalgubeol-daero, Dalseo-gu,

Daegu 42601, Korea

Tel: $+82-53-258-7824$

Fax: $+82-53-258-4875$

E-mail: yejeeshim@dsmc.or.kr
Background Pilocytic astrocytoma (PA) is a brain tumor that is relatively more common in children and young adults.

Methods We retrospectively reviewed the medical records of patients with PA treated at a single center between 1988 and 2018.

Results We included 31 subjects with PA. The median age at diagnosis was 13.4 years, and the median follow-up duration was 9.9 years. The total PA group had a 10-year disease-specific survival (DSS) rate of 92.6\% [95\% confidence interval (Cl), 82.6-100] and 10-year progression-free survival (PFS) rate of $52.8 \%$ (95\% Cl, 32.0-73.6). In patients aged <20 years, tumors were more likely to be located in sites in which gross total tumor resection (GTR) was impossible. No statistically significant difference in 10-year DSS was found between the GTR (100\%) and non-GTR (89.7\%; 95\% CI, 76.2-100; $p=0.374)$ groups. However, a statistically significant difference in 10-year PFS was found between the GTR (100\%) and non-GTR groups (30.7\%; 95\% Cl, 8.6-52.8; $p=0.012)$. In the non-GTR group, no statistically significant difference in 10-year DSS was found between the patients who received immediate additional chemotherapy and/or radiotherapy (Add-Tx group, 92.9\%; 95\% Cl, 79.4-100) and the non-Add-Tx group (83.3\%; 95\% Cl, 53.5-100; $p=0.577$ ). No statistically significant difference in 10year PFS was found between the Add-Tx group (28.9\%; 95\% Cl, 1.7-56.1) and non-Add-Tx group (33.3\%; 95\% Cl, 0-70.9; $p=0.706)$.

Conclusion The PFS of the patients with PA in our study depended only on the degree of surgical excision associated with tumor location. This study is limited by its small number of patients and retrospective nature. A multicenter and prospective study is necessary to confirm these findings.

Key Words Glioma; Survivors; Child; Adolescent; Prognosis.

\section{INTRODUCTION}

Pilocytic astrocytoma (PA) is brain tumor that is relatively more common in children and young adults. PA is a World Health Organization grade I neoplasm with an expected benign course and a 10-year survival rate exceeding 95\% [1,2]. In Korea, single-center data about the prognosis of PA in a metropolitan area [3] and in the Honam area [4] have been re-

This is an Open Access article distributed under the terms of the Creative Commons Attribution Non-Commercial License (https://creativecommons.org/licenses/by-nc/4.0) which permits unrestricted non-commercial use, distribution, and reproduction in any medium, provided the original work is properly cited.

Copyright $(\underset{2019}{ } 20$ The Korean Brain Tumor Society, The Korean Society for NeuroOncology, and The Korean Society for Pediatric Neuro-Oncology ported. However, no data are available on survival and prognosis in patients with PA in the Yeungnam area. Thus, we retrospectively reviewed the long-term survival and prognosis of patients with PA in a single center in the Yeungnam region.

\section{MATERIALS AND METHODS}

\section{Subjects and ethics statement}

Patients who were diagnosed with PA and followed up at Keimyung University Dongsan Medical Center from January 1988 to December 2018 were enrolled. We pathologically confirmed primary brain tumors through biopsy; thus, optic or brainstem gliomas that were not available for biopsy were ex- 
cluded. This study was approved by the Institutional Review Board of Keimyung University Dongsan Medical Center (no. 2017-11-075). Data from the patients' medical records, including those pertaining to age at diagnosis, sex, initial symptoms or signs, tumor site, treatment modality, survival rate, and complications, were collected and retrospectively reviewed.

\section{Definitions}

Gross total tumor resection (GTR) was defined when no obvious visible tumor was found on brain imaging performed soon after the operation. If a portion of the tumor was still found on a brain imaging scan after surgery, it was defined as a subtotal tumor resection (STR) when the residual tumor was $<50 \%$ and as partial tumor resection (PTR) when the residual tumor was $\geq 50 \%$ according to the neurosurgeon's judgment [4]. After surgical resection and/or postoperative radiotherapy or chemotherapy, the development of new lesions or the increase of tumor size from that of the original residual tumor was defined as progression. Disease-specific survival (DSS) is defined as the proportion of people who died of PA progression; patients who died of medical conditions other than PA were not included in DSS.

\section{Statistical analyses}

To compare the ratio of the variables between the 2 groups, the $\chi^{2}$ test was used. The 10 -year probabilities of overall survival (OS), DSS, and progression-free survival (PFS) were estimated with the Kaplan-Meier method using the log-rank test. $p$ values $<0.05$ indicated statistical significance. All statistical analyses were performed using SPSS v23.0 software (IBM Corp., Armonk, NY, USA). The 95\% confidence intervals (CIs) were calculated using the mean values and standard errors.

\section{RESULTS}

\section{Subjects' characteristics and clinical manifestations}

During the study period, 1,017 patients with pathologically confirmed primary tumors that originated from the central
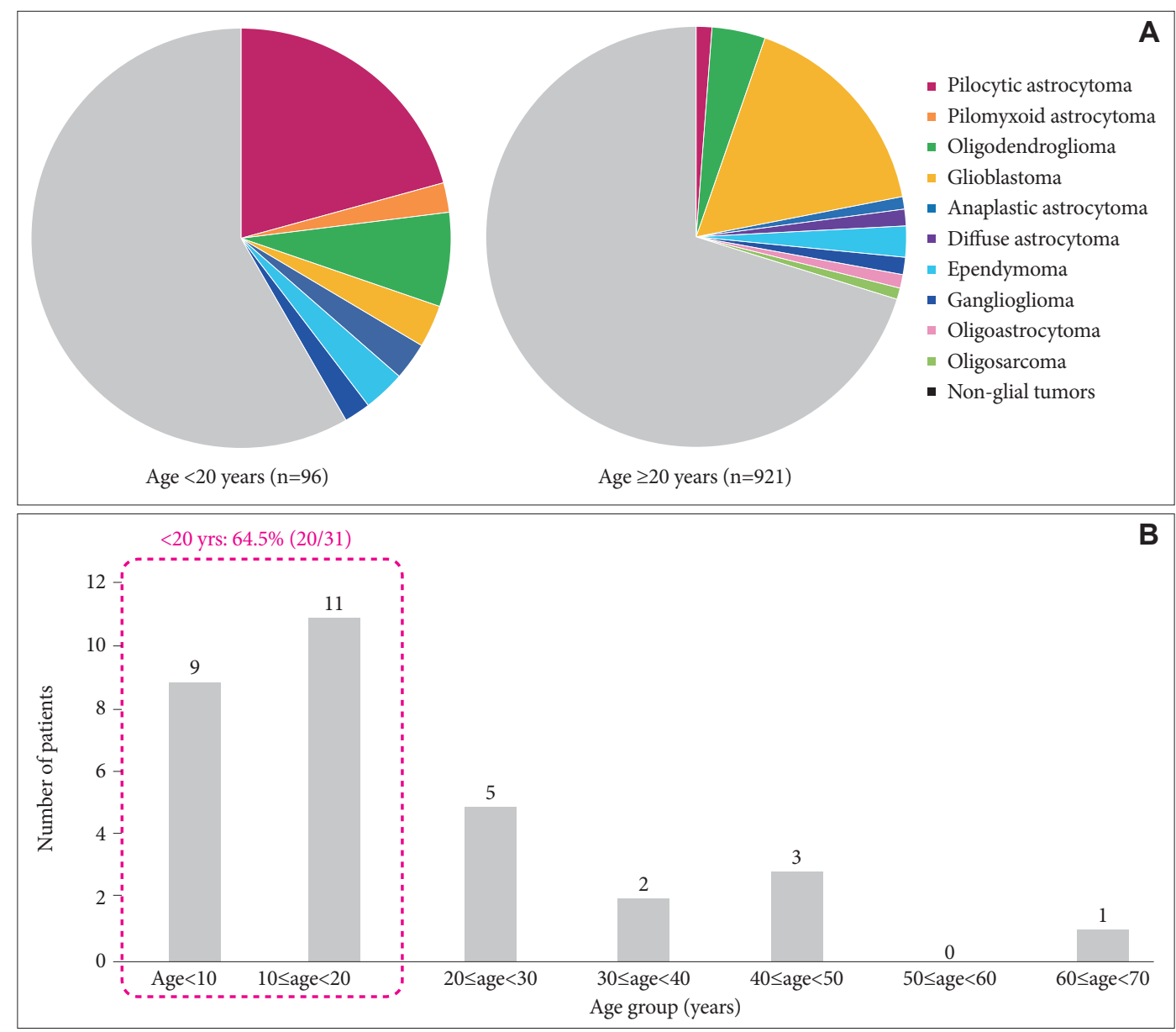

Fig. 1. The ratio of glial tumors between age groups in a single medical center between1988 and 2018. A: A total of 1,017 patients had pathologically confirmed primary brain tumors; of them, 314 had glial tumors. Pilocytic astrocytoma occurred more frequently in patients aged $<20$ years $(20.8 \%)$ than in those aged $\geq 20$ years $(1.2 \% ; p<0.001)$. B: The ratio of pilocytic astrocytoma among the patients aged $<20$ years was $64.5 \%$. 
nervous system were treated at our center. Among them, 314 had glial brain tumors; 155 , glioblastoma; 46, oligodendroglioma; 31, PA; 2, pilomyxoid astrocytoma; 24, ependymoma; 15 , ganglioglioma; 13 , diffuse astrocytoma; 12 , anaplastic astrocytoma; 9, oligoastrocytoma; and 7, gliosarcoma. The ratio of PA to the total number of primary brain tumors was higher in the patients aged $<20$ years [20.8\% (20/96)] than in those aged $\geq 20$ years [1.2\% (11/921); $p<0.001]$. The ratios of the respective disease groups by patient age are shown in Fig. 1A.

Thirty-one subjects had PA (male-to-female ratio, 15:16). Among them, one had neurofibromatosis 1 confirmed by genetic testing. The median age at diagnosis was 13.4 years (range, 0.6-67.0 years). The median follow-up duration was 9.9 years (range, $0.1-30.2$ years). The PA distribution by age is shown in Fig. 1B. The patients with PA had symptoms or signs that included headache (7), seizure (6), vomiting (6), mental change (6), visual disturbance (6), gait disturbance (6) or hemiparesis (5). The tumor sites were the cerebrum (11), cerebellum (8), optic chiasm and hypothalamus (7), brain stem (2), basal ganglia (2), and ventricle (1). The baseline characteristics of the subjects with PA are summarized in Table 1.

\section{Treatment modalities}

Among the 31 patients with PA, 10 underwent GTR; 14, STR; 3, PTR; and 4, biopsy. In the non-GTR group ( $\mathrm{n}=21)$, additional immediate chemotherapy and/or radiotherapy were administered to 15 patients (Add-Tx group): chemotherapy was administered to 10 patients and radiotherapy was administered to 7 patients ( 2 patients received both). In the patients aged $<20$ years, tumors were more likely to be located in sites where GTR could not be performed, including the optic chiasm and hypothalamus (7), brain stem (2), and basal ganglia (2). The respective treatment modalities for the patients with PA are described in Table 2. The chemotherapy regimens included: CCG9952A (carboplatin and vincristine) (7), Ghim's protocol [5] (vinblastine, etoposide, 5 -fluorouracil, and cyclophosphamide) (1), and POG9031 (cisplatin and etoposide) (2). The median radiotherapy dose was 5,400 cGy (range, 4,500-6,000 cGy).

\section{Survival rate}

In the total PA group, the 10-year DSS, OS, and PFS were 92.6\% (95\% CI, 82.6-100), 88.4\% (95\% CI, 66.4-98.6), and $52.8 \%$ (95\% CI, 32.0-73.6), respectively (Fig. 2). Three patients died of causes other than brain tumor progression, 1 patient died of infection and septic shock at 13.9 years after the diagnosis of PA, 1 patient died of a psychological problem and suicide at 10.2 years after diagnosis, and 1 patient died of an unknown cause of death at 3.6 years after diagnosis. No statistically significant difference in 10-year DSS was found among the GTR
Table 1. Baseline characteristics of patients with pilocytic astrocytoma treated at a single center

\begin{tabular}{|c|c|}
\hline Characteristic & Value \\
\hline Age at diagnosis (years), median (range) & $13.4(0.6-67.0)$ \\
\hline Sex (male to female ratio) & $15: 16$ \\
\hline Underlying anomaly (NF1 to non-NF1 ratio) & $1: 30$ \\
\hline Follow-up duration (years), median (range) & $9.9(0.1-30.2)$ \\
\hline \multicolumn{2}{|l|}{ Symptoms or signs at diagnosis, $\mathrm{n}(\%)$} \\
\hline Headache & $7(22.6)$ \\
\hline Seizure & $6(19.4)$ \\
\hline Vomiting & $6(19.4)$ \\
\hline Mental change & $6(19.4)$ \\
\hline Visual disturbance & $6(19.4)$ \\
\hline Gait disturbance & $6(19.4)$ \\
\hline Hemiparesis & $5(16.1)$ \\
\hline Sensory change & $3(9.7)$ \\
\hline Facial palsy & $3(9.7)$ \\
\hline Male precocious puberty & $2(6.5)$ \\
\hline Dysarthria & $2(6.5)$ \\
\hline Nystagmus & $1(3.2)$ \\
\hline Dysmetria & $1(3.2)$ \\
\hline \multicolumn{2}{|l|}{ Site, n (\%) } \\
\hline \multicolumn{2}{|l|}{ Supratentorial tumor } \\
\hline Cerebrum & $11(35.5)$ \\
\hline Optic chiasm and hypothalamus & $7(22.6)$ \\
\hline Basal ganglia & $2(6.5)$ \\
\hline Ventricle & $1(3.1)$ \\
\hline \multicolumn{2}{|l|}{ Infratentorial tumor } \\
\hline Cerebellum & $8(25.8)$ \\
\hline Brain stem & $2(6.5)$ \\
\hline
\end{tabular}

NF1, neurofibromatosis 1

(100\%), STR (92.9\%; 95\% CI, 79.4-100), PTR (100\%), and biopsy (75\%; 95\% CI, 32.5-100, $p=0.120$ ) (Fig. 3A) groups. However, a significant statistical difference in 10-year PFS was found among the GTR (100\%), STR (43.7\%; 95\% CI, 15.571.9), PTR (0\%), and biopsy groups (0\%; $p<0.001)$ (Fig. 3B). No statistically significant difference in 10-year DSS was found between the GTR (100\%) and non-GTR $(89.7 \%$; 95\% CI, 76.2$100, p=0.374$ ) (Fig. 3C) groups. However, a statistically significant difference in 10-year PFS was found between the GTR (100\%) and non-GTR (30.7\%, 95\% CI, 8.6-52.8; $p=0.012$ ) (Fig. 3D) groups. No statistically significant difference in 10-year DSS was found between the <20-year (94.7\%; 95\% CI, 84.7100 ) and $\geq 20$-year (88.9\%; 95\% CI, 68.3-100; $p=0.634)$ age groups. No statistically significant difference in 10 -year PFS was found between the <20-year (37.9\%; 95\% CI, 12.2-63.6) and $\geq 20$-year age group (65.6\%; 95\% CI, 33.5-97.7; $p=0.432$ ). In the non-GTR group, no statistically significant difference in 10year DSS was found between the Add-Tx (92.9\%; 95\% CI, 79.4-100) and non-Add-Tx (83.3\%; 95\% CI, 53.5-100; 
Table 2. Treatment modalities for patients with pilocytic astrocytoma treated at a single center

\begin{tabular}{|c|c|}
\hline Tumor site (n) & Treatment (n) \\
\hline \multicolumn{2}{|l|}{ Aged $<20$ years $(n=20)$} \\
\hline $\begin{array}{l}\text { Optic chiasm } \\
\text { and hypothalamus (7) }\end{array}$ & $\begin{array}{l}\text { STR }(3) \rightarrow \text { CT or RT }(3) ; \\
\text { PTR }(2) \rightarrow \text { CT or RT }(2) ; \\
\text { Biopsy }(2) \rightarrow \text { CT or RT }(2)\end{array}$ \\
\hline Cerebellum (4) & $\operatorname{GTR}(2) ; \operatorname{STR}(2) \rightarrow$ CT or RT $(1)$ \\
\hline Cerebrum (5) & $\operatorname{GTR}(2) ; \operatorname{STR}(3) \rightarrow$ CT or RT $(1)$ \\
\hline Brain stem (2) & $\begin{array}{l}\text { PTR }(1) \rightarrow \text { CT or RT }(1) ; \\
\text { Biopsy }(1) \rightarrow \text { CT or RT }(1)\end{array}$ \\
\hline Basal ganglia (2) & $\begin{array}{l}\text { STR }(1) \rightarrow \text { CT or RT }(1) \\
\text { Biopsy }(1) \rightarrow \text { CT or RT }(1)\end{array}$ \\
\hline \multicolumn{2}{|l|}{ Aged $\geq 20$ years $(n=11)$} \\
\hline Cerebrum (6) & GTR (3); STR (3) $\rightarrow$ CT or RT (2) \\
\hline Cerebellum (4) & GTR (3); STR (1) \\
\hline Ventricle (1) & $\operatorname{STR}(1)$ \\
\hline
\end{tabular}

GTR, gross total tumor resection; STR, subtotal tumor resection; PTR, partial tumor resection; CT, chemotherapy; RT, radiotherapy

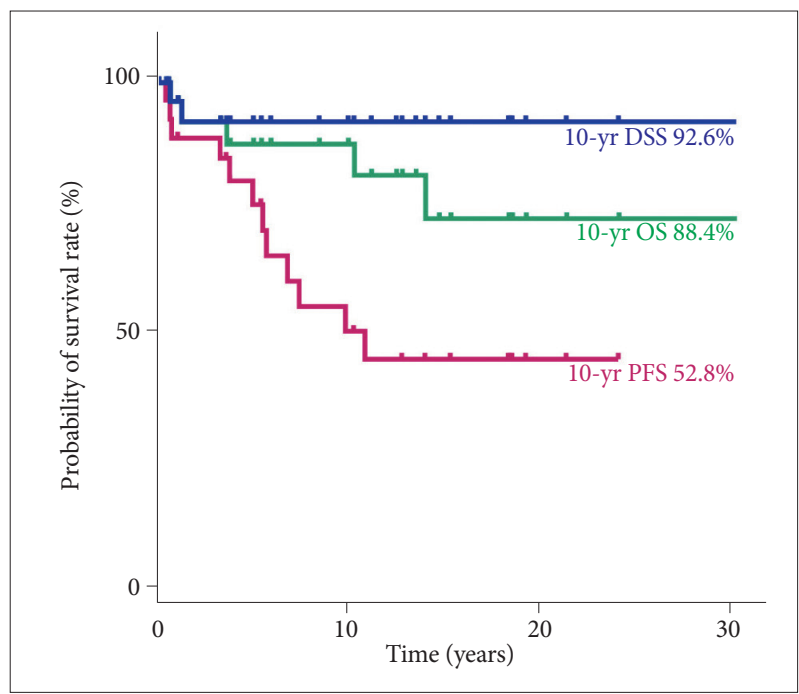

Fig. 2. The survival rate of the patients with pilocytic astrocytoma in a single center. The 10 -year DSS, OS, and PFS were $92.6 \%$ $(95 \% \mathrm{Cl}, 82.6-100), 88.4 \%(95 \% \mathrm{Cl}, 66.4-98.6)$, and $52.8 \%$ $(95 \% \mathrm{Cl}, 32.0-73.6)$. DSS, disease-specific survival; OS, overall survival; PFS, progression-free survival; $\mathrm{Cl}$, confidence interval.

$p=0.577)$ groups. No statistically significant difference in 10year PFS was found between the Add-Tx (28.9\%; 95\% CI, 1.756.1 ) and non-Add-Tx (33.3\%; 95\% CI, $0-70.9 ; p=0.706)$ groups.

\section{Sequelae and complications}

The survivors had neurological complications, including seizure (13), headache (4), dysarthria (4), ataxia (4), paralysis (3), fine motor failure (2), dizziness (1), and facial palsy (1).
The survivors also had psychiatric complications, including alcohol dependence (3), nervous personality (2), isolation (1), major depressive disorder (1), self-harm (1), suicide (1), somatizing syndrome (1), attention deficit hyperactivity disorder (1), hallucination (1), bulimia nervosa (1), inappropriate behavior (1), and violent behavior (1). They also had endocrine problems, including precocious puberty (4), growth hormone deficiency (4), panhypopituitarism (3), and obesity (1).

\section{DISCUSSION}

PA is the most common brain tumor with a low-grade histology in the first 2 decades of life [6]. PAs can be found along the neuraxis, including the optic chiasm, hypothalamus, cerebral hemisphere, and brain stem; $80 \%$ of cases are found in the cerebellum [7]. In our study, PA was also the most common primary brain tumor in patients aged $<20$ years. However, optic chiasmatic-hypothalamic and brain stem tumors were relatively more frequent in the patients aged $<20$ years in our study.

Surgical resection is the standard initial approach to managing PA, and GTR is desirable. PA is generally a benign tumor that features excellent outcomes with GTR [8]. If removal is complete, no further treatment is necessary. However, a PA that arises in the optic pathway, brain stem, and hypothalamus is not usually amenable to GTR. Some factors, including PTR, tumor location, or invasion of the surrounding structures, are associated with a worse prognosis [8]. In a prospective nonrandomized study of childhood low-grade glioma (LGG) performed by The Children's Cancer Group and Pediatric Oncology Group, the 5-year PFS was 90\% with GTR and 45-65\% with any volume of residual tumor $(p<0.001)$ [9]. Tumor site was also associated with PFS. Chiasmatic-hypothalamic or midline tumors, for which GTR is difficult to perform, showed lower PFS than cerebellar or cerebral tumors [9].

If resection is incomplete, the need for immediate additional therapy such as chemotherapy or radiotherapy remains controversial among clinicians [10]. Some evidence indicates that additional radiotherapy may prolong PFS but has little impact on OS [10]. In the European Organization for Research and Treatment of Cancer study of 22,845 patients, 311 patients with LGG aged $\geq 16$ years were randomized to receive postoperative observation or immediate radiotherapy [11]. The irradiated patients showed a significantly improved 5 -year PFS, but no significant difference in 5-year OS was observed between the 2 arms [11]. Thus, the authors recommend additional treatment only after re-progression of the tumor and on the basis of the premise that OS will be unaffected regardless of whether PFS significantly improves [6,7].

In fact, radiotherapy is generally reserved for cases in 


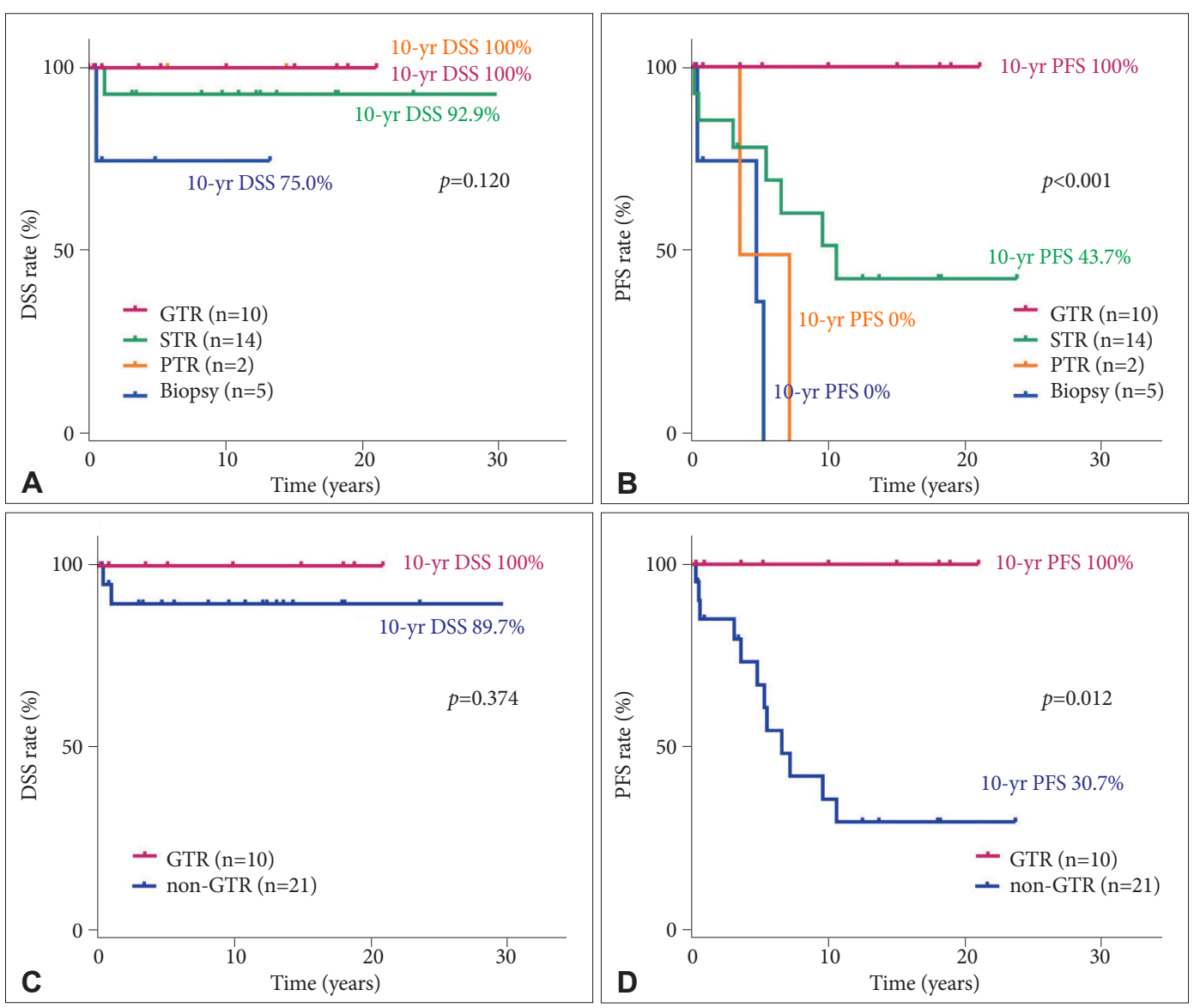

Fig. 3. Survival rates of the patients with pilocytic astrocytoma according to resection success. No statistically significant difference in 10year DSS was found between the GTR and non-GTR groups (A and C). However, a statistically significant difference in 10-year PFS was observed between the GTR and non-GTR groups (B and D). DSS, disease-specific survival; PFS, progression-free survival; GTR, gross total resection; STR, subtotal tumor resection; PTR, partial tumor resection.

which multiple chemotherapy treatments have failed in unresectable symptomatic tumors because of its toxicity. To minimize the risk of late sequelae of brain radiation exposure, the use of chemotherapy to delay radiation exposure in young children has become widespread in pediatric cases. In a recent study that analyzed the efficacy of CV (vincristine and carboplatin) in 113 children with LGG, an overall response to chemotherapy was observed in $92 \%$ of the children, while the median time to progression was 22.5 months in $42 \%$ of the children [12]. On the basis of the COG A9952 results, the use of either TPCV [carboplatin, procarbazine, CCNU (lomustine), and vincristine] or CV provides adequate tumor control that allows a radiotherapy delay [13].

In a single-center study in the Korean capital area that included 91 pediatric patients with PA, the 10-year PFS was higher in those who received postoperative additional treatment, including radiotherapy or chemotherapy, than in those who were only observed [3]. However, no statistically significant difference in 10-year PFS was found between the Add-Tx and non-Add-Tx groups in our study. We suggest that this is be- cause of the retrospective single-center design of our study with fewer cases. On the other hand, no significant difference in 10year DSS was observed between the GTR and non-GTR groups in our study. Thus, we suggest that immediate additional treatment such as radiotherapy or chemotherapy would play a role in the treatment of our patients. In a single-center study in the Korean Honam area that included approximately $39 \mathrm{pa}-$ tients with PA, tumor progression in adult patients with PA followed a more aggressive clinical course than that in pediatric patients with PA [4]. However, no statistically significant difference in 10-year DSS or PFS was found between the $<20$ - and $\geq 20$-years age groups in our study, possibly because the tumors in the patients aged $<20$ years in our study were relatively more commonly located in areas where surgery is not likely to completely remove them (optic chiasmatic-hypothalamic or brain stem tumors).

The Epidemiology and End Results database reported on the long-term outcomes of 4,040 pediatric patients with LGG [14]. The 20 -year OS was $87 \%$, and the 20 -year cumulative incidence of death due to LGG glioma was $12 \%$. Prognostic 
factors included patient age, tumor histology and grade, primary tumor site, radiation dose, and degree of surgical resection on univariate analysis [14]. In the multivariate analysis, the greatest mortality risk was associated with the use of radiation [14]. Thus, the strategies for pediatric LGG should aim for disease control with emphasis on minimizing longterm toxicities associated with treatment [14]. Neurocognitive and endocrine dysfunctions are considerable factors that could be related with tumor progression or treatment-related toxicity [15]. Our study also showed several late sequelae and neurological, psychological, endocrinologic, and other complications, although it remains unclear whether these symptoms originated from tumor location or treatment modality.

In conclusion, the patients with PA in our institute also showed a high survival rate similar to those in other institutions. The PFS of the patients with PA in our study only depended on the degree of surgical excision associated with the tumor location. As mentioned earlier, our study has some limitations. As it was retrospective and conducted in a single institute with a small number of patients, whether additional treatments for PA could increase the PFS rate is unclear. Despite these limitations, we think that this study is valuable because it is the first to investigate the long-term survival and prognosis of patients with PA in the Korean Yeungnam region. As shown in this study, although the survival rate was high in these patients, several medical sequelae and complications were experienced over the long term. Therefore, attention should be paid to managing the complications encountered by these long-term survivors. In addition, a Korean multicenter study is needed to evaluate the accurate prognosis of patients with PA.

\section{Conflicts of Interest}

The authors have no potential conflicts of interest.

\section{Acknowledgments}

This research was supported by Basic Science Research Program through the National Research Foundation of Korea (NRF) funded by the Ministry of Science and ICT (grant number: NRF-2017R1C1B5016851).

\section{REFERENCES}

1. Ostrom QT, Gittleman H, Liao P, et al. CBTRUS statistical report: primary brain and other central nervous system tumors diagnosed in the United States in 2010-2014. Neuro Oncol 2017;19(suppl_5):v1-88.

2. Ostrom QT, Gittleman H, Xu J, et al. CBTRUS statistical report: primary brain and other central nervous system tumors diagnosed in the United States in 2009-2013. Neuro Oncol 2016;18(suppl_5):v1-75.

3. Jung SY, Jin SL, Hahn SM, et al. Radiation or chemotherapy rather than observation may be a better modality after subtotal resection for pilocytic astrocytoma in children. Clin Pediatr Hematol Oncol 2014;21:95-103.

4. Ryu HH, Jung TY, Lee GJ, et al. Differences in the clinical courses of pediatric and adult pilocytic astrocytomas with progression: a singleinstitution study. Childs Nerv Syst 2015;31:2063-9.

5. Cho BK, Jung HL, Ghim TT, et al. KSPNO protocol for glioma. Korean J Pediatr Hematol Oncol 2005;12:244-85.

6. Banerjee A, Nicolaides T. Low-gade gliomas. In: Gupta N, Banerjee A, Haas-Kogan DA, editors. Pediatric CNS tumors. 3rd ed. Heidelberg: Springer; 2017.p. 1-35.

7. Dirven CM, Mooij JJ, Molenaar WM. Cerebellar pilocytic astrocytoma: a treatment protocol based upon analysis of 73 cases and a review of the literature. Childs Nerv Syst 1997;13:17-23.

8. Fernandez C, Figarella-Branger D, Girard N, et al. Pilocytic astrocytomas in children: prognostic factors--a retrospective study of 80 cases. Neurosurgery 2003;53:544-53; discussion 554-5.

9. Shaw EG, Wisoff JH. Prospective clinical trials of intracranial lowgrade glioma in adults and children. Neuro Oncol 2003;5:153-60.

10. Pollack IF, Claassen D, al-Shboul Q, Janosky JE, Deutsch M. Lowgrade gliomas of the cerebral hemispheres in children: an analysis of 71 cases. J Neurosurg 1995;82:536-47.

11. Karim AB, Afra D, Cornu P, et al. Randomized trial on the efficacy of radiotherapy for cerebral low-grade glioma in the adult: European Organization for Research and Treatment of Cancer Study 22845 with the Medical Research Council study BRO4: an interim analysis. Int J Radiat Oncol Biol Phys 2002;52:316-24.

12. Gnekow AK, Falkenstein F, von Hornstein S, et al. Long-term followup of the multicenter, multidisciplinary treatment study HITLGG-1996 for low-grade glioma in children and adolescents of the German Speaking Society of Pediatric Oncology and Hematology. Neuro Oncol 2012;14:1265-84.

13. Ater JL, Zhou T, Holmes E, et al. Randomized study of two chemotherapy regimens for treatment of low-grade glioma in young children: a report from the Children's Oncology Group. J Clin Oncol 2012;30:2641-7.

14. Bandopadhayay P, Bergthold G, London WB, et al. Long-term outcome of 4,040 children diagnosed with pediatric low-grade gliomas: an analysis of the Surveillance Epidemiology and End Results (SEER) database. Pediatr Blood Cancer 2014;61:1173-9.

15. Kortmann RD, Timmermann B, Taylor RE, et al. Current and future strategies in radiotherapy of childhood low-grade glioma of the brain. Part II: treatment-related late toxicity. Strahlenther Onkol 2003;179:585-97. 